\title{
COVID-19 Spatiotemporal Hotspots and Prediction Based on Wavelet and Neural Network
}

\author{
Neda Kaffash Charandabi and Amir Gholami
}

In this chapter, a global model of the COVID-19 is proposed to determine the important periods of each country, prediction of confirmed cases, and discover spatiotemporal hot/cold spots. The importance of the COVID-19 periods is assessed for each country and the most important periods are selected as time series prediction delays and temporal neighborhood steps of spatiotemporal analysis. The COVID-19 cases are predicted based on wavelet and neural network with an average RMSEIQR of 0.974 for all countries. Finally, the hot/cold spot maps are prepared by the specified temporal neighborhood steps and the patterns are identified. More than $61 \%$ of the earth's surface is surrounded by COVID-19 hot spots.

\subsection{Introduction}

The novel Coronavirus Disease 2019 (COVID-19) has spread all over the world since it first appeared in Wuhan, the capital city of Hubei province, on 31 December 2019. The number of cases is well above 35 million and the number of deaths is more than 1 million till 5 October 2020, according to the World Health Organization (WHO) situation reports [1]. The WHO announced COVID-19 as the sixth the world's public health concern on January 30, 2020. It is transmitted via human-to-human droplets or direct contact, and the mean incubation period for infection has been estimated to be 6.4 days [2]. However, there is little information about this new virus, researchers in different fields are working towards discovering an appropriate solution to this global issue [2]. One of the new approaches to better management of epidemics is the use of spatiotemporal analyses. These analyses are important tools for preventing and reducing the spread of disease, with the potential to detect trends and critical points of the disease outbreaks.

The Geographic Information System (GIS) is an analytical tool for collecting, editing, managing, and processing of spatial data. GIS is also used as a platform for spatiotemporal analyses by integrating temporal data with location and attribute data. Conventional GIS analyses are very useful in the identification of spatiotemporal patterns and clusters. However, it needs to be combined with robust algorithms to predict time series. Neural network algorithms are among the most common families of non-parametric methods that can be used for predicting epidemic peaks. But they alone are not enough to predict variable, nonlinear, and uncertain issues. Wavelet is used as one of the most powerful methods in signal processing and time series analysis. It is possible to predict complex time series with high accuracy by combining the wavelet with neural networks [3].

In recent years, more researchers have concentrated on predicting epidemic outbreaks. Al-Ahmadi et al. investigated MERS-COV data in Saudi Arabia from 2012 to 2019. The disease was analyzed by extracting spatial, temporal, seasonal, and spatial-temporal clusters [4]. Mongkolsawat 
and Kamchai identified the critical areas of Avian influenza in Thailand. This study highlighted the use of GIS to investigate the prevalence and identification of critical areas in different epidemics [5]. Li et al. described the spatial and temporal characteristics of human H7N9 virus infections in China using data from 2013 to 2017 and ArcMap ${ }^{\mathrm{TM}} 10.2$ along with SaTScan [6]. Zhu et al. used the multi-channel Long Short-Term Memory (LSTM) to predict influenza in China. The neural network training process was performed with number of legal influenza cases and outbreaks, affected cases with different ages, Chinese patent cold medicines, other cold medicines, temperature, rainfall, air pressure, and relative humidity for nine years in nine regions of China [7]. Venna et al. applied data-driven machine learning to predict flu based on environmental factors. Meteorological, proximity, and influenza data from 1997 to 2016 were analyzed using the LSTM deep learning model [8]. Spataru utilized the ArcMap ${ }^{\mathrm{TM}} 10.2$ "space-time pattern mining" tool to analyze polio disease. He had extracted space-time clusters and critical locations of the disease based on Mann-Kendall and Getis-Ord Gi* statistic [9]. According to the previous studies, the use of spatiotemporal analysis has been very useful in studying epidemics. However, spatiotemporal clustering and time series analysis had been carried out separately in previous researches.

Numerous studies have started in the field of treatment and management of the COVID-19. Guan et al. studied data from 1099 patients of 552 hospitals in 30 different provinces. Based on their findings over the first 2 months, the COVID-19 has spread around the world with different conditions and symptoms [10]. Lai et al. studied COVID-19 patient data in countries around the world until February 11 and used graphs and maps to examine their patient numbers and specific symptoms [2]. Kuniya predicted the epidemic peak of Coronavirus using the SEIR model in Japan. In his study, early middle summer was known as the peak of the COVID-19 in Japan, so forecasting for all countries based on suitable methods seems to be necessary [11]. Al-qaness et al. proposed a method for forecasting confirmed cases of the COVID-19 in China based on an Adaptive Neuro-Fuzzy Inference System (ANFIS) using an enhanced Flower Pollination Algorithm (FPA) along with the Salp Swarm Algorithm (SSA) [12]. Chakraborty and Ghosh forecasted Coronavirus cases base on wavelet and AutoRegressive Integrated Moving Average model (ARIMA) for Canada, France, India, South Korea, and the UK [13]. Tamang et al. predicted Covid-19 cases based on an Artificial Neural Network (ANN) curve fitting technique [14]. These predictions have been made in line with the current trend of rising cases in different countries and the patterns of change in China and South Korea for one week. The reviewed articles provided an example of researches in the field of space and statistics. In these studies, less attentions have been paid to both the SpatioTemporal Hot/Cold Spot Analysis (STHCSA) and the Coronavirus pandemic time series prediction. Previous research has only been performed in one or some countries and has not been a global model. Periods are not predicted for each country, and the accuracy of the training data is assessed, while the accuracy of the test and train data indicates the actual accuracy of the prediction.

\subsection{Materials and Methods}

In this chapter, a combined model of wavelet and neural network was used to predict COVID-19 cases based on data reported by WHO, due to its ability to complex time series prediction [3]. Also, its hot/cold spots were identified by specified important periods and features. The theoretical foundations of the methods used in this research were described in this section.

\subsubsection{Wavelet transforms}

Wavelet transform is a mathematical approach to the decomposition of data into a variety of frequency components. It can extract special patterns hidden in a huge amount of data. The wavelet transform can be used to analyze non-stationary time series data at several different frequencies. Wavelet transforms are generally divided into the Continuous Wavelet Transformation (CWT) and Discrete Wavelets Transformation (DWT) [15]. The DWT and CWT are the wavelet transform 
implementation using discrete and arbitrary sets of the wavelet scales, respectively. They can be used to decompose wavelets, process signals, extract features, and denoise noisy signals. There are many types of mother wavelets that can be used for wavelet transforms. The different mother wavelets that are used to examine the same signal will yield varying results. Therefore, different mother wavelet types of CWT and DWT were examined to select best of them for capturing the multiscale features of signals $[16,17]$.

\subsubsection{CWT}

The CWT is an important method for assessing non-stationary signals and providing a number of signal information, such as time, frequency, scale, and local signal correlation. The CWT is used to decompose a signal into small wavelets that are highly localized in time [18]. It generally used short-time Fourier transform for decomposes a signal into unlimited length sines and cosines, basically eliminating all time-localization information and replacing it with time-frequency signal representation that provides very strong time and frequency localization. The wavelet function is defined as Eq. (17.1) The basic functions of the CWT are scaled and shifted versions of a function called the time-localized mother wavelet $\Psi(t)$. Eq. (17.2) express wavelet transform that is the convolution of time series data and wavelets $[18,19]$.

$$
\begin{gathered}
\int_{-\infty}^{\infty} \Psi(t) \mathrm{dt}=0 . \\
F(a, b)=\frac{1}{\sqrt{a}} \int_{-\infty}^{\infty} f(t) \Psi\left(\frac{t-b}{a}\right) \mathrm{dt} .
\end{gathered}
$$

Where a and $\mathrm{b}$ are the scale and shift parameters, $t$ is the time, $f(t)$ is the data, $\Psi(t)$ is the mother wavelet, and $F(a, b)$ is the time-scale representation of the signal. Several mother wavelets are available for the CWT, including Mexican hat wavelet, analytic Morlet wavelet, generalized Morse wavelet, Bump wavelet, and so on. The Morlet is a wavelet composed mainly of an exponential function multiplied by a Gaussian window. This wavelet is closely related to human perception and demonstrates good performance in analyzing the periodicity of local signals. Generalized Morse wavelets are a family of analytical wavelets with two parameters, symmetry and time-bandwidth of the product. Bump wavelet has a larger variance in time and smaller variance in frequency. Each of these wavelets has different parameters for studying different behaviors and properties [18, 20].

\subsubsection{DWT}

The DWT is a powerful time series analysis tool used to break down the original time series into different components, each of which can produce meaningful information from the original data. The DWT can be decomposed the signal into low and high frequencies. The low and high frequencies are also called as approximation and detail coefficients. Commonly the approximation is decomposed to a higher level after the first level. The DWT is used to reduce time series data in order to save storage space while losing a small amount of detailed information. The last coefficient of approximation and a few of the high-level detail coefficients are typically chosen for preservation that are the only coefficients needed for perfect reconstruction. Hence, The DWT is known as a lossless transformation, whereby transformed domain data can collectively rebuild the original data $[16,21]$.

The DWT has several mother wavelet families, such as Daubechies, Coiflets, Symlets, Fejér-Korovkin, discrete Meyer, Biorthogonal, and reverse Biorthogonal, which each of them has different orders. The Daubechies family is an orthonormal wavelet, which makes the analysis of wavelets possible in discrete time. The first order Daubechies (db1) wavelet resembles a simple step function and the higher-order Daubechies functions (db2, db3, db4, etc.) are not easy to define with an analytical expression. The order of this function shows the number of vanishing moments or the number of zero wavelet moments. The Coiflet (coif) wavelet family is more symmetrical and has more vanishing moments than the Daubechies wavelets. Symlets' (sym) properties are similar to Daubechies, that are near-symmetric and have the least asymmetry. Fejér-Korovkin (fk) wavelet family minimizes the gap between the ideal since lowpass filter and the valid scaling filter. The 
discrete Meyer (dmey) wavelet family is defined in the frequency domain. The Biorthogonal (bio) and reverse Biorthogonal (rbio) families use separate wavelet and scaling functions for the analysis, synthesis, and vice versa [22].

\subsubsection{Neural networks}

ANNs are human brain-inspired methods that consist of a large number of simple and highly interconnected computing elements and use them as a huge data processing system. ANN-based methods are very useful for prediction problems. Three main parts of the ANN are the input, hidden, and output layers [23]. Different models of the ANNs were introduced to solve specific problems. In this research Multi Layer Perceptron (MLP) was used as a class of feedforward neural networks which is an efficient and popular algorithm. The MLP is a type of supervised learning algorithms that uses backpropagation to train. The MLP contains a number of layers, neurons, weights, and transfer functions. Transfer functions are used to aggregate the input neurons with different weights to the output where the neuron is a link between the layers. First, the input neurons are multiplied by their respective weights, then the output neuron is summed up and determined via the transfer function. Tansig is one of the widely used transfer functions that has been selected for this research. This function makes MLP networks so powerful, because of the ability to represent nonlinear functions [23, 24].

Finally, normalized Root Means Square Error by the Inter-Quartile Ranges (RMSEIQR) was used to evaluate the accuracy of the prediction results of the proposed model because it is suitable for comparing the different values obtained for various countries with a different population and confirmed cases. The RMSEIQR is an interquartile range of the RMSE that normalizes values and is less sensitive to extreme values (outliers) than the RMSE. The RMSEIQR is defined as Eq. (17.3) where $\hat{y}_{t}$ and $y_{t}$ are predicted and observed values over T time and IQR is the difference of quartile functions. In other words, the RMSEIQR calculates by dividing the RMSE into the IQR [25].

$$
R M S E I Q R=\frac{\sqrt{\sum_{t=1}^{T}\left(\hat{y}_{t}-y_{t}\right)^{2}}}{I Q R \sqrt{T}} .
$$

\subsubsection{Hot/Cold spot analysis}

Spatiotemporal analysis was used to identified hot/cold spots by specified important periods and features in previous steps. STHCSA is an important component of spatiotemporal analysis since location and time are two critical aspects of important events such as the Coronavirus epidemic. The outputs of such analyses can provide useful information to guide the activities aimed at preventing, detecting, and responding to pandemic problems [26]. There are various methods for spatiotemporal analysis. In this study, Mann-Kendall test was used to detect trends of data and Getis-Ord Gi* statistic was used to identify hot/cold spots.

Network Common Data Form (NetCDF) is a file format to store multi-dimensional scientific data such as temperature, humidity, disease, and crime. The NetCDF cube is generated using the COVID-19 $\mathrm{x}, \mathrm{y}$, and time data as $\mathrm{x}, \mathrm{y}$, and $\mathrm{z}$ axes. It summarizes a collection of points into a NetCDF by aggregating them into space-time bins. The Mann-Kendall p-values and z-scores show the statistical significance of the trend in a hot spot (spatial clusters of high values) or cold spot (spatial clusters of low values) at a location. A positive or negative z-score indicates an upward or downward trend respectively $[9,27]$. Then, the pattern in the spatiotemporal data was identified with Getis-Ord Gi* statistic based on neighborhood distance and neighborhood time step. The Getis-Ord $\mathrm{Gi}^{*}$ statistic is calculated for each bin as follows [28, 29]:

$$
\begin{gathered}
\bar{x}=\frac{\sum_{j=1}^{n} x_{j}}{n} . \\
s=\sqrt{\frac{\sum_{j=1}^{n} x_{j}^{2}}{n}-\bar{x}^{2} .}
\end{gathered}
$$




$$
G_{i}^{*}=\frac{\sum_{j=1}^{n} w_{i j} x_{j}-\bar{x} \sum_{j=1}^{n} w_{i j}}{s \sqrt{\frac{n \sum_{j=1}^{n} w_{i j}^{2}-\left(\sum_{j=1}^{n} w_{i j}\right)^{2}}{n-1}}} .
$$

Where $x_{j}$ is the value of feature $x$ at location $j, n$ is the number of data and $w_{i j}$ is the element of the weight matrix. The $G_{i}^{*}$ is recorded as a z-score for each variable in the dataset. The more intense the clustering of high values or hot spots are, the larger positive z-scores become and the more intense the clustering of low values or cold spots are, the smaller negative z-scores get. Based on p-values and z-scores of this statistic, 17 pattern types include: no pattern detected, new hot spot, consecutive hot spot, intensifying hot spot, persistent hot spot, diminishing hot spot, sporadic hot spot, oscillating hot spot, historical hot spot, new cold spot, consecutive cold spot, intensifying cold spot, persistent cold spot, diminishing Cold Spot, sporadic cold spot, oscillating cold spot and historical cold spot are extracted [9].

\subsection{Results of Proposed Model}

In this chapter, the confirmed cases of the COVID-19 in all countries around the world based on WHO reports until June 24 were used as input signals. The proposed model of this research was implemented in four steps: 1) identification of significant periods using CWT, 2) extraction of effective features using DWT, 3) prediction of cases with neural network based on the outputs of steps 1 and 2, and 4) extraction of hot/cold spot patterns. The procedure and the results of each step are discussed below.

\subsubsection{Identification of significant periods using CWT}

The COVID-19 epidemic is a highly contagious disease and may have a long incubation period, which means that patients understand it late and therefore quickly infects others without regarding social distance. Therefore, many people get the disease in a short period of time, and after an incubation period, the number of patients suddenly increases significantly. This trend is easily understood from the COVID-19 confirmed cases data and important periods can be deduced from them.

The confirmed cases of the COVID-19 were divided into train and test data as input signals. The data for the last 7 days (17 to June 24, 2020) of each country was selected as the test data. Due to the high outbreak of COVID-19 and its long and short incubation periods, signal behavior can be analyzed, its relative and absolute maxima can be identified, and significant periods can be recognized for each country. The CWT was used to extract significant periods and these periods were used as delays in the prediction step. The significant frequency (reverse of the period), the date of its occurrence, and the magnitude were calculated for each country and shown on 3D plot (Figure 17.1). Based on the 3D surfaces, relative or local maxima were identified to determine the peak of important periods. For each country, periods, magnitudes, and dates of its occurrence have been identified by the CWT. The aim of this chapter is to investigate the longer and shorter serial intervals of COVID-19 that are associated with the incubation periods. The period of incubation for COVID-19 is the time between virus exposure and the onset of symptoms, which can be contagious to some people during this period. Understanding the incubation period enables health authorities to establish more effective quarantine systems for people suspected of carrying the virus as a means of monitoring and preventing the spread of the virus [1].Based on WHO reports, the incubation period for seasonal influenza, SARS, and MERS was typically around 2-14 days. Various studies have been conducted to determine the period of COVID-19. Qun Li et al. has found the incubation period to be 5.2 days on average [30] but in another research these periods were between 3 and 7 days, up to 14 days [31]. In addition, other cases with 19 and 27 incubation periods were reported by Hubei province. These periods were between 2-14 and 2-10 days based on the United States' CDC and WHO reports, respectively [1]. 

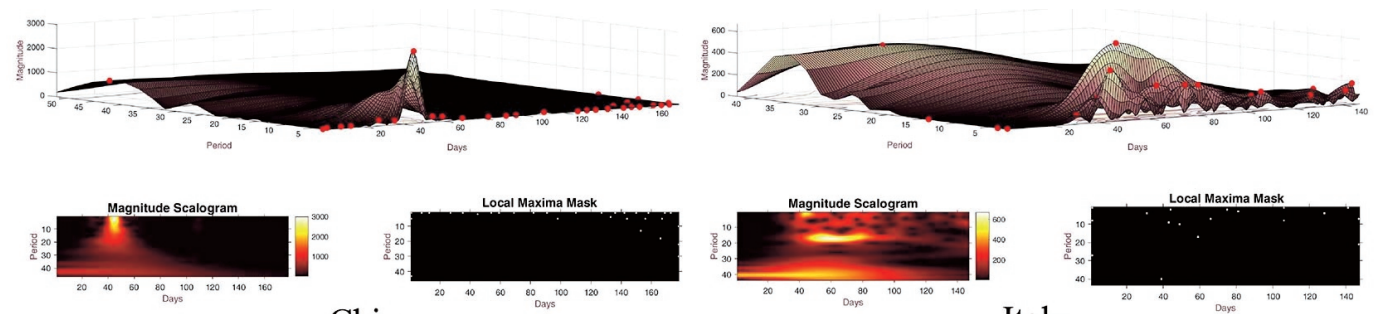

China

Italy
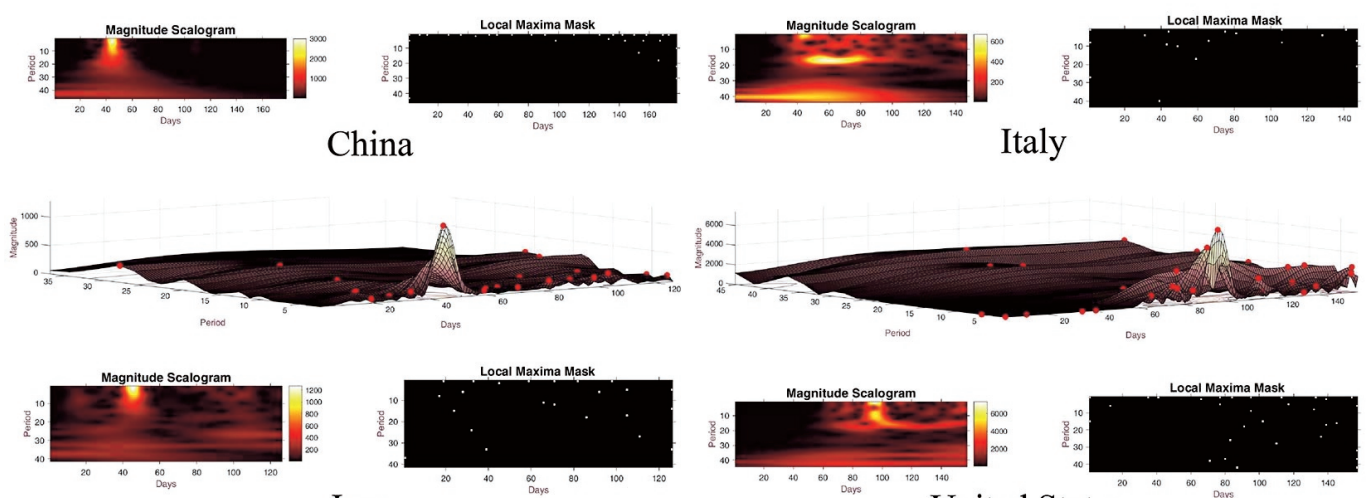

Iran

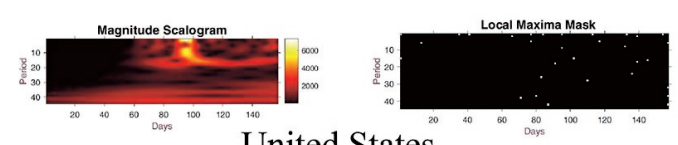

United States
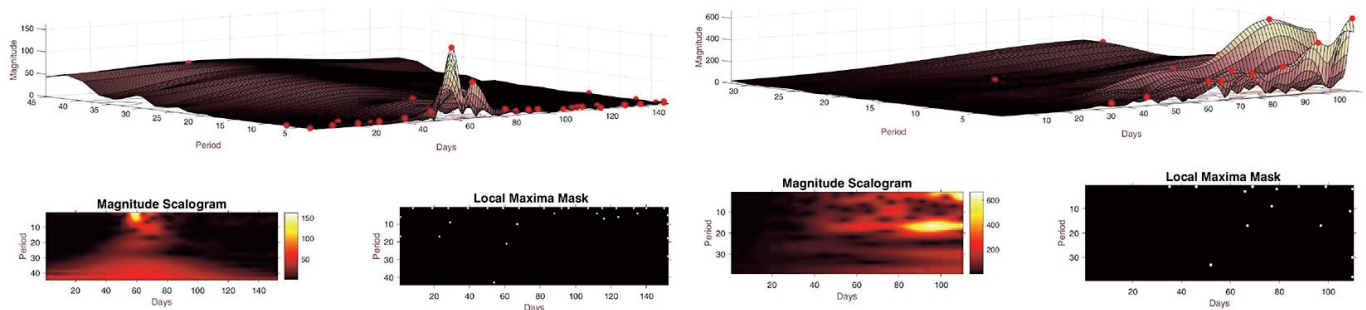

Australia

Mexico

\section{FIGURE 17.1}

The CWT results for COVID-19 confirmed cases of six countries.

Previous research, based on clinical trials, identified incubation periods for some countries using restricted cases. While in this chapter, significant periods (the longer and shorter serial intervals of COVID-19) for all countries have been identified based on CWT and the input signal that their accuracy depends on the accuracy of WHO reports. They can considerably represent incubation periods. For example, according to the results of this research, significant periods for China were $2.72,4.41,5.43,16.46,17.64,40.53$, and 65.85 days which 2.71 and 40.53 days were more important than others. Also, COVID-19 important periods for Italy were about 3, 4, 7, 9, 38, 47, and 53 days while these periods for Iran were about $3,4,6,12,14,22$, and 47 days. It was done for each country and the important periods of each country were identified. For example, periods in China, Italy, Iran, the United States, Australia, and Mexico are shown in Figure 17.1. The results indicate that the three days period was important in most countries. Periods of 2-14 days and periods greater than 25,40 , and 65 days were also very notable. In particular, the (2-7)-day period was very important as one of the results of this research that was emphasized in previous clinical studies. The range and frequency of important periods that were extracted for all countries are shown in Figure 17.2. Comparison of CWT results and clinical studies reveals that the research outcome is highly accurate, which can be easily derived from the reported COVID-19 cases for all countries.

Also, due to the different results of the use of different mother wavelet functions, each CWT function was examined for each country and the function that had better RMSE was selected for each country. For instance, the best CWT function for the United States, China, Italy, Australia, and Mexico was bump and morlet (amor) for Iran. 


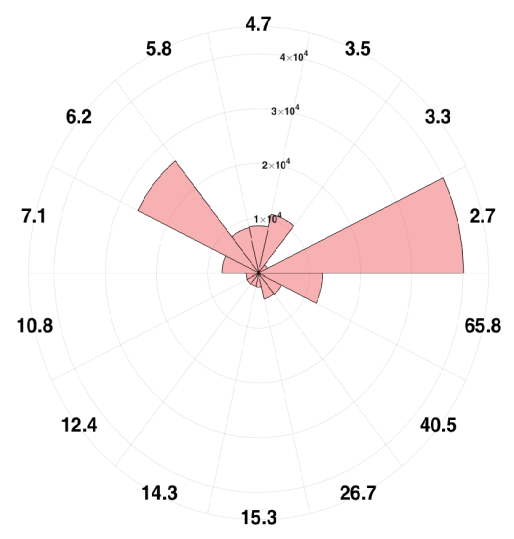

\section{FIGURE 17.2}

The range and frequency of important periods of all countries around the world based on CWT results.

\subsubsection{Extraction of effective features using DWT}

After identifying the significant periods of each country in the previous step, they can be used as a time series prediction delay. In other words, previous days which had an impact on the number of cases each day were determined by these periods for the neural network. The input signal (or the probability density function of patients) alone is not sufficient to predict accurately and it is better to extract the features from the original signal. Because the confirmed number of patients with COVID-19 is affected by many different factors that are not easily identifiable. For example, cultural factors in the timely referral of patients, a number of tests in each country, the integrity of governments in reporting cases, and so on, have a direct impact on the number of reported cases, although they are almost impossible to characterize due to lack of data. Therefore, it is better to extract the features of the daily COVID-19 reports from the input data signals. Artificial intelligence can consider features without identifying the name and type of features.

The DWT is one of the most popular tools for feature extraction. In this study, the five signal levels for each country were calculated by DWT. In the first step, the DWT was applied to the input signal and the approximation and detail were extracted. In the next step, this transformation was applied to the approximation extracted from the previous level. This process continued for five levels and finally, five approximations and details were obtained. All details of the levels and the approximation of the last level were used as ANN features. For example, the result of applying DWT to Iran confirmed cases is shown in Figure 17.3 with D1, D2, D3, D4, D5, and A5. The DWT has been done for all countries, and the features of each country have been identified for entry into the ANN.

Also in DWT, different mother wavelet functions with different orders were evaluated and the function that led to the lowest RMSE for each country was selected. For example, the best DWT functions for the United States, China, Italy, Iran, Australia, and Mexico were fk22, coif1, d6, sym9, db1, and coif5, respectively. Figure 17.4 shows the results of CWT and DWT implementation of different mother wavelet functions for each country. For 213 countries and territories around the world, 3 mother wavelet families of CWT and 6 mother wavelet families with 40 types of DWT functions were implemented. For example, in the 152nd country, Russia, bump and rbio were chosen as the appropriate mother wavelet functions. The numerical proportions of the use of CWT and DWT mother wavelet functions are also shown in the left and right pie charts of Figure 17.4. The results show that morse and bior1.1 were the most desirable functions among the CWT and DWT functions which were used $41.95 \%$ and $16.0976 \%$, respectively. 

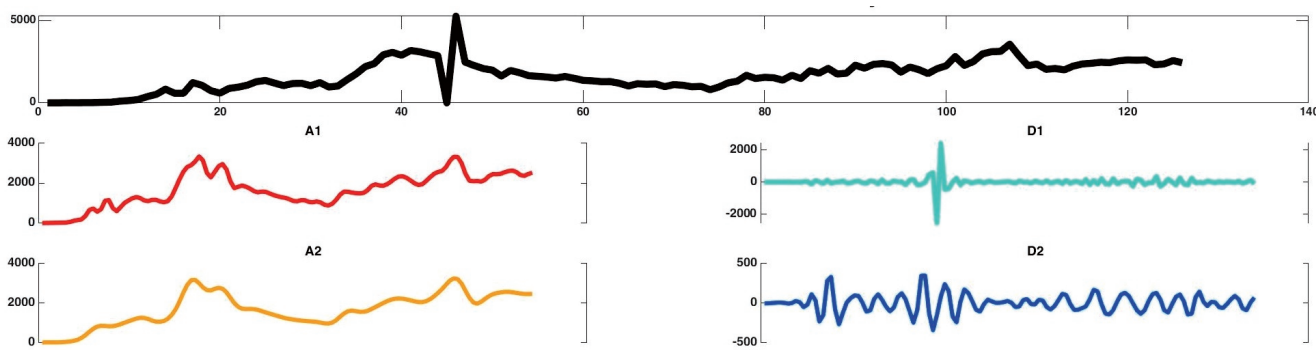

A3
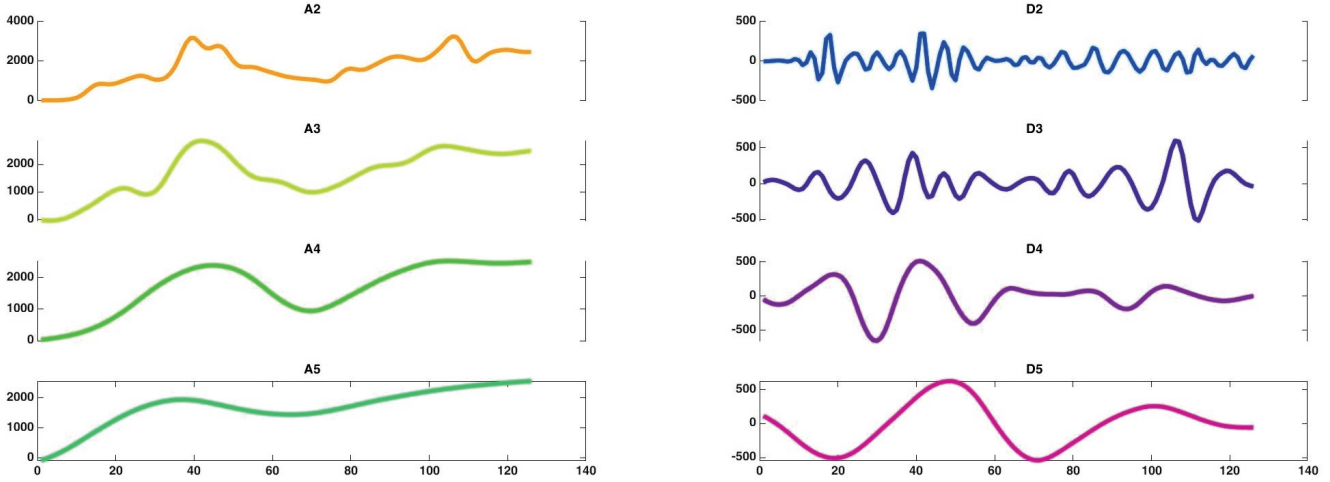

\section{FIGURE 17.3}

The DWT results for COVID-19 confirmed cases of Iran.

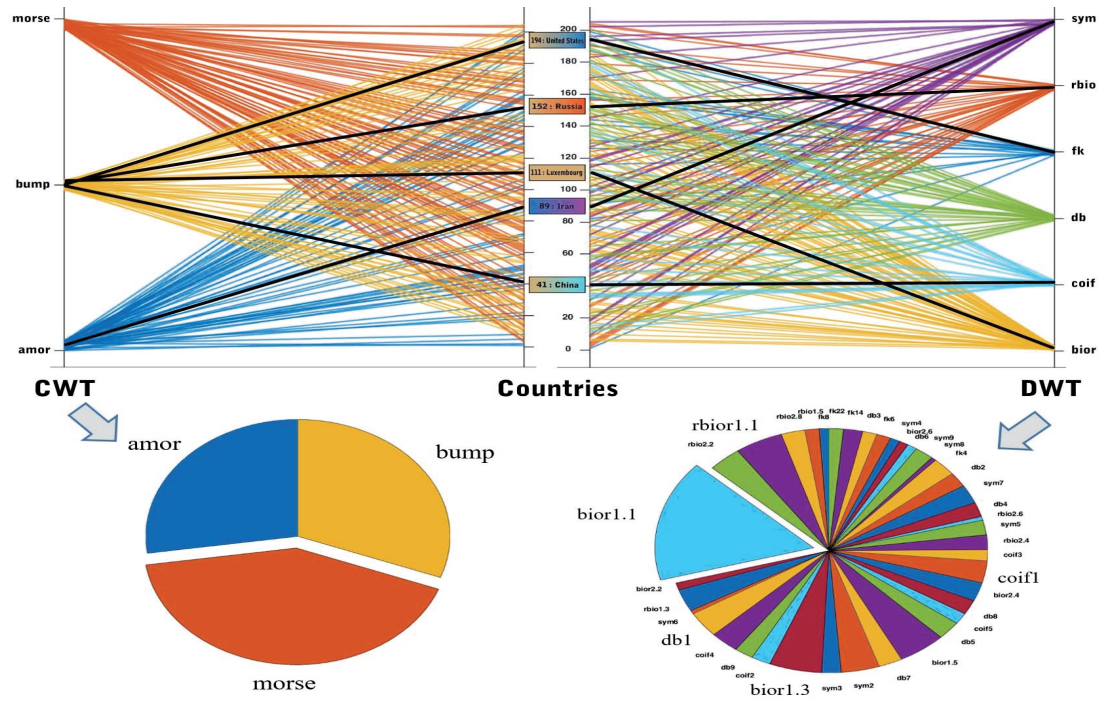

\section{FIGURE 17.4}

Comparison of CWT and DWT mother wavelet functions.

\subsubsection{Prediction model based on a neural network}

The extracted features must be delayed according to the detected periods before entering the ANN. Thus, the number of input features was $(n s+k)^{*} n p$, where $\mathrm{k}$ is the number of input signals $(\mathrm{k}=1)$, $\mathrm{ns}$ is the number of sub-series, and $\mathrm{np}$ is the number of important periods for each country. In this study, ns was six because there were five levels of detail (D1, D2, D3, D4, and D5) and one level of approximation (A5). For example, the most important periods for Australia were about 3, 4, 9, and 41 days. They applied to the main signal and all subseries (k, D1, D2, D3, D4, D5, and A1) and 
$4^{*}(6+1)$ features (28 features) were prepared as the network inputs. A single-layer perceptron neural network with a sigmoid transfer function has been implemented for each country. The advantages of the MLP network and the reason for choosing the sigmoid function were described in the materials and methods section. As a generalization error is so important in predicting time series, an attempt has been made to select a network size as small as possible to prevent overfitting.

After selecting the features and creating an optimal network, based on the confirmed cases of the COVID-19 up to June 17, the networks were trained and, for the last seven days, the forecast was carried out as test data. The prediction models have been developed for all countries and the results for the six countries: the United States, China, Italy, Iran, Australia, and Mexico are presented in Figure 17.5. In these plots, the horizontal axis is the number of the days, the vertical axis is the number of patients, the red dashed line is the observed cases, the continuous blue line is the predicted cases, and the white and gray parts are the train and test sections of data, respectively. These plots show the spread of the disease, its peaks, sudden increases, and the results of predictions in each country. The RMSE of train and test data has been calculated to evaluate the results of the prediction. The best and worst RMSE of predictions among these six countries were for Australia and the United States with 4.23 and 2264.92 for test data as well as 61.6852 and 3631.14 for train data. For a more detailed review, the test and train RMSE values, CWT and DWT mother wavelet functions, and the significant periods in 14 countries that are more affected by the COVID-19 are shown in Table 17.1.

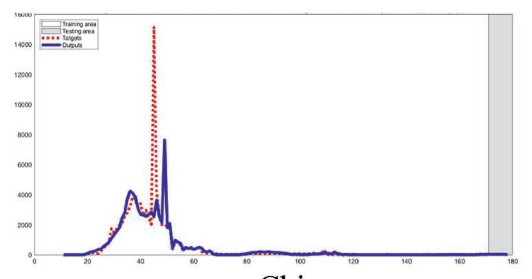

China
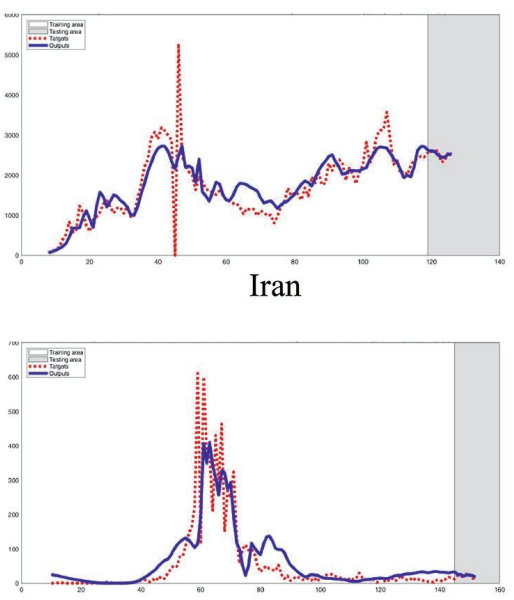

Australia
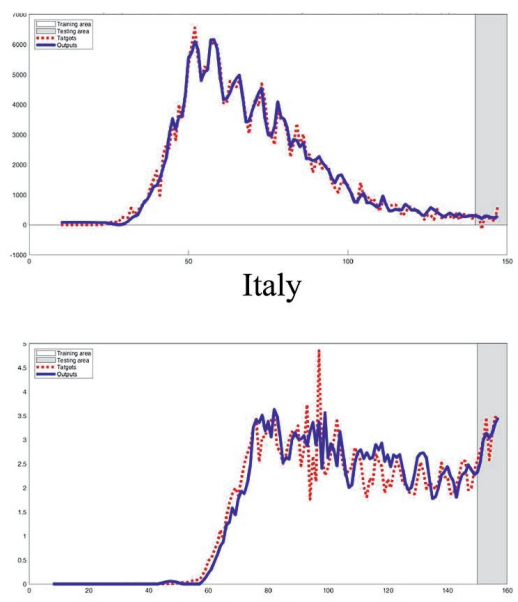

United States

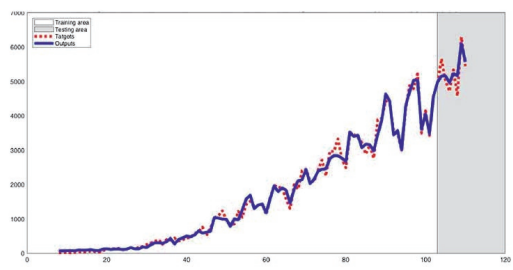

Mexico

\section{FIGURE 17.5}

Prediction results of the COVID-19 confirmed cases for six countries. 
TABLE 17.1

List of detailed information about inputs and results of prediction in 14 counties.

\begin{tabular}{|c|c|c|c|c|c|}
\hline Country & $\begin{array}{l}\text { Train } \\
\text { RMSE }\end{array}$ & $\begin{array}{l}\text { Test } \\
\text { RMSE }\end{array}$ & CWT & $\overline{\mathrm{DWT}}$ & Periods \\
\hline Australia & 61.6852 & 4.23448 & bump & db1 & $\begin{array}{l}2.72-4.41 \\
9.46-40.54\end{array}$ \\
\hline Brazil & 2094.0814 & 6800.2734 & amor & rbio1.1 & $\begin{array}{l}2.72-3.34 \\
3.58-5.43 \\
6.69-7.68 \\
21.72-43.45\end{array}$ \\
\hline Canada & 154.2365 & 30.5101 & morse & bior1.5 & $\begin{array}{l}2.72-4.12 \\
8.23-13.37 \\
26.74-53.49\end{array}$ \\
\hline China & 1101.6565 & 8.70 & bump & coif1 & $\begin{array}{l}2.72-4.41 \\
5.43-16.46 \\
17.64-40.53 \\
65.85\end{array}$ \\
\hline Germany & 356.2333 & 81.2454 & amor & coif3 & $\begin{array}{l}2.72-6.69- \\
10.8613 .37- \\
15.3653 .49\end{array}$ \\
\hline India & 208.8657 & 1329.085 & morse & bior 1.5 & $\begin{array}{l}2.72-2.91 \\
3.58-5.07 \\
6.24-7.17 \\
8.82-20.27\end{array}$ \\
\hline Iran & 456.87 & 82.5656 & amor & sym9 & $\begin{array}{l}2.72-3.84 \\
6.24-11.64 \\
14.33-21.72 \\
46.56\end{array}$ \\
\hline Italy & 219.5788 & 329.0488 & bump & db6 & $\begin{array}{l}2.72-3.58 \\
6.69-9.46 \\
37.82-46.56 \\
53.49\end{array}$ \\
\hline Mexico & 135.006 & 329.0488 & bump & coif5 & $\begin{array}{l}2.72-2.91 \\
3.84-4.12 \\
6.69-13.37 \\
17.64-30.72\end{array}$ \\
\hline Russia & 162.9836 & 145.0177 & bump & rbio2.4 & $\begin{array}{l}2.72-3.84 \\
6.69-18.91 \\
20.27-30.72 \\
53.49\end{array}$ \\
\hline South Korea & 625.2626 & 12.3547 & morse & sym9 & $\begin{array}{l}2.72-3.58 \\
4.12-4.73 \\
5.07-7.17 \\
28.66-43.45\end{array}$ \\
\hline Spain & 411.7153 & 83.6592 & amor & $\mathrm{db} 2$ & $\begin{array}{l}2.72-3.34 \\
5.82-6.24 \\
7.17-8.23 \\
13.37-49.91\end{array}$ \\
\hline Turkey & 77.9651 & 297.4952 & morse & $\mathrm{db} 5$ & $\begin{array}{l}2.72-3.84 \\
4.41-5.82 \\
7.68-9.46 \\
18.91-37.82\end{array}$ \\
\hline United States & 3631.1439 & 2264.9258 & bump & fk22 & $\begin{array}{l}2.72-3.12 \\
4.12-6.69 \\
768-28.66 \\
43.45-57.33\end{array}$ \\
\hline
\end{tabular}




\subsubsection{Extraction of hot/cold spot patterns}

Finally, the COVID-19 pattern maps were prepared with the STHCSA at different periods. The neighborhood time step must be specified in the preparation of this map. The neighborhood time step is the number of time-step intervals to be used in the neighborhood of analysis. This value specifies which features are evaluated together to determine local clustering in space-time cube [28]. Hot/Cold spots of the COVID-19 confirmed cases in the world countries with one-day neighbors until March 21, 2020 are presented in Figure 17.6.

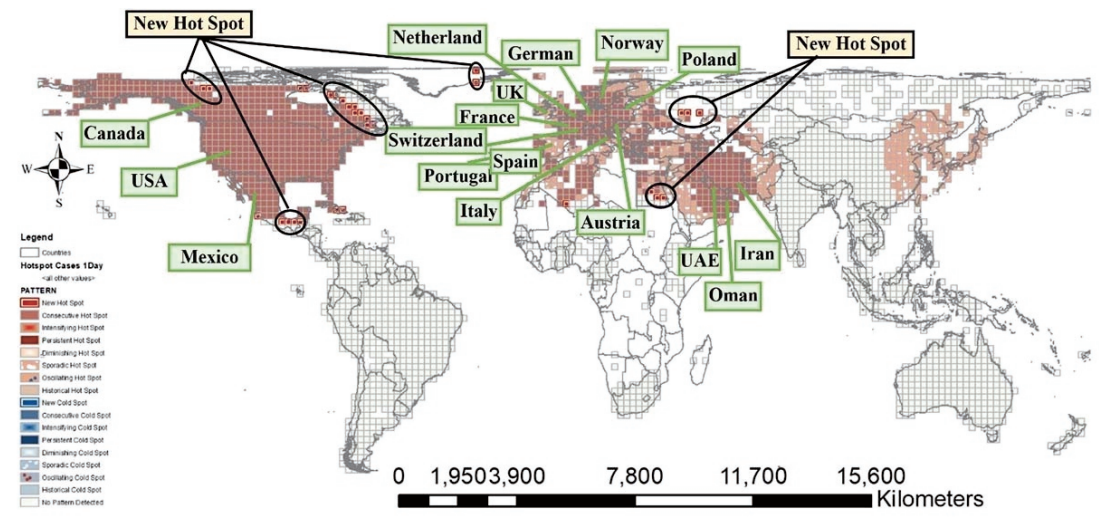

\section{FIGURE 17.6}

The STHCSA results for confirmed cases with a day temporal neighborhood steps.

The spatiotemporal patterns were identified with respect to the number of confirmed cases, the area, the spatial and temporal neighbors of each country. Countries such as the USA, Italy, Germany, France, Portugal, Mexico, Iran, Poland, Austria, Switzerland, Netherlands, and UAE have consecutive hot spots according to the results in Figure 17.6. The consecutive hot spot is a location with a single continuous run of statistically notable hot spot bins in the final time-step intervals. New hot spots in countries like Canada, Mexico, Cuba, Egypt, and Russia were also identified. Other countries were recognized as the oscillating hot spot or without a pattern. The new hot spot area is a location that is statistically significant for the final time step and has never been a statistically significant hot spot before. The oscillating hot spot is a region with high temporal oscillation patterns. With the temporal neighborhood increasing to three days, parts of Australia and Venezuela had been identified as historic cold spots and western China as oscillating cold spots. New hot spots had been detected in Brazil, Mexico, Russia, and Australia based on data until March 21, and after this date, there was a huge increase especially in Brazil and Russia.

Then, the spatiotemporal patterns were re-examined and shown in Figure 17.7 by entering all the data until June 24. The temporal neighborhood steps were entered as 3, 6, and 66 days according to the important periods extracted for the whole world and results are shown in Figure 17.7.a, 17.7.b, and 17.7.c, respectively. With increasing temporal neighborhood steps from 3 to 66 days, oscillation and consecutive hot spots have increased by $14 \%$ for Asian and European regions and about $10 \%$ for the United States. Countries like India, Brazil, Mexico, and Russia have become oscillation and consecutive hot spots. According to the results, many parts of the southern hemisphere have been identified as persistent and intensifying cold spots due to their low confirmed cases and cold spots have also increased by $20 \%$. 


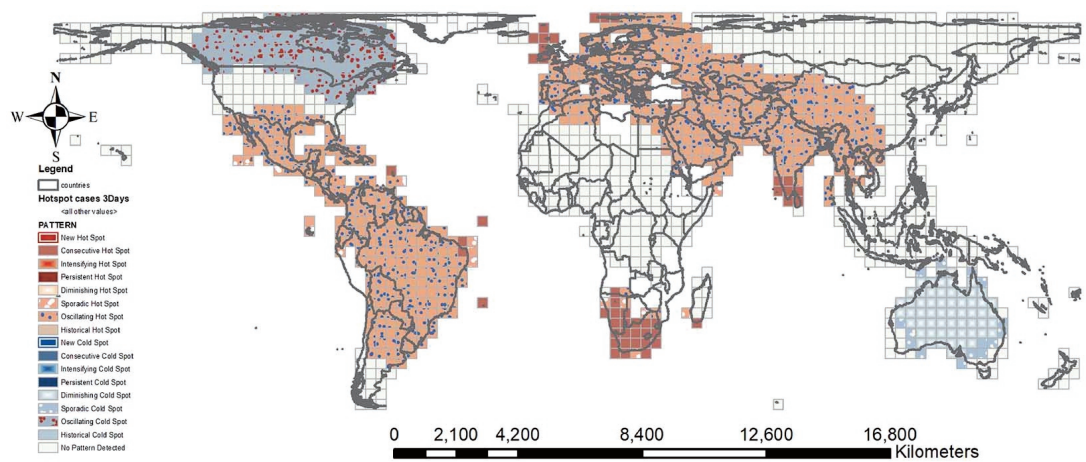

a) Results for confirmed cases with three days of temporal neighborhood steps

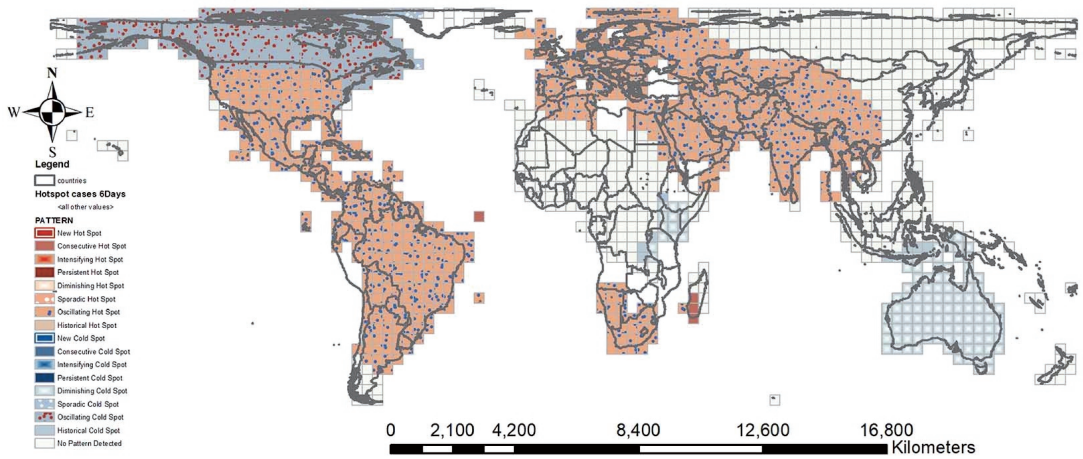

b) Results for confirmed cases with six days of temporal neighborhood steps

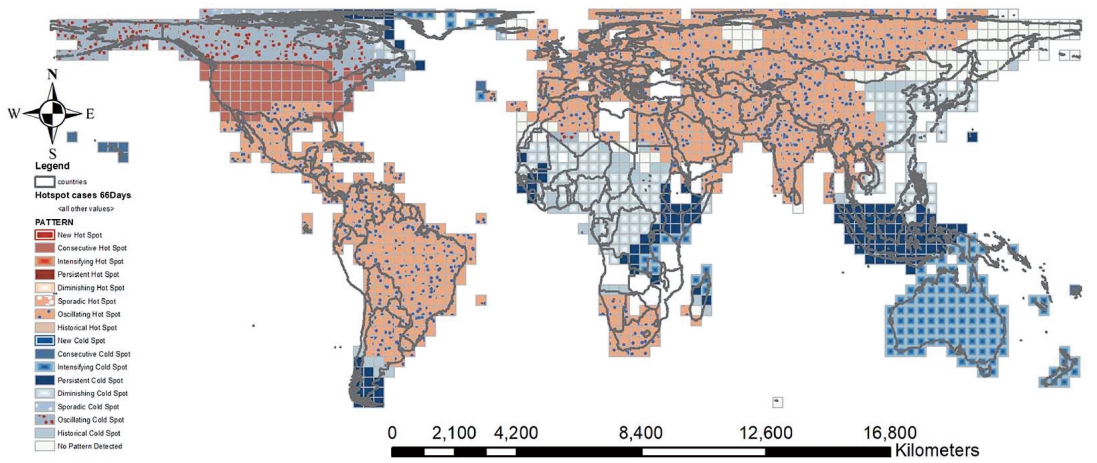

c) Results for confirmed cases with sixty six days of temporal neighborhood steps

\section{FIGURE 17.7}

The STHCSA maps.

\subsection{Discussion}

The (2-7)-day period had a large magnitude in the results of this research, which had also been highlighted in clinical research. Results also highlighted periods greater than 25,40 , and 65 days which should be considered, whereas clinical trials had recorded only periods about 25 days. It 
seems that if the time series data are recorded correctly in terms of number and time, the higher the rate of infection and the incubation period of the diseases, the more possible it will be to extract important periods from them. The 3,6, and 66-day periods with a large magnitude are shown in Figure 17.8. The size of the symbols indicates the normalized magnitude of the periods and their colors show the importance of the classes that are classified by the k-means.

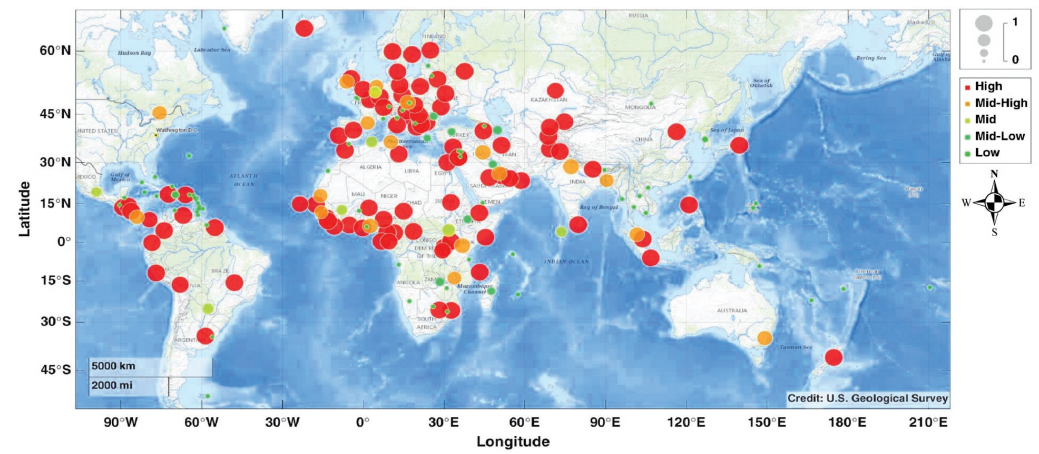

a) The importance of three-days period for each country

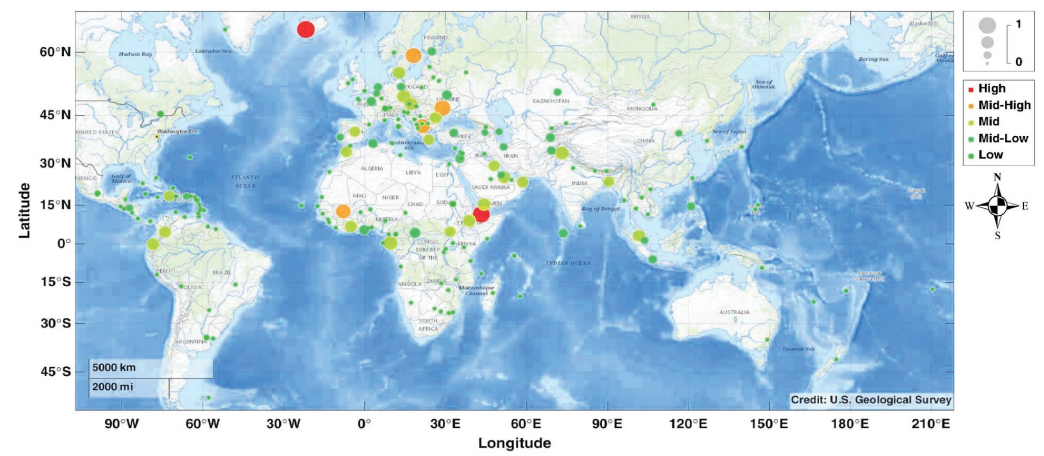

b) The importance of six-days period for each country

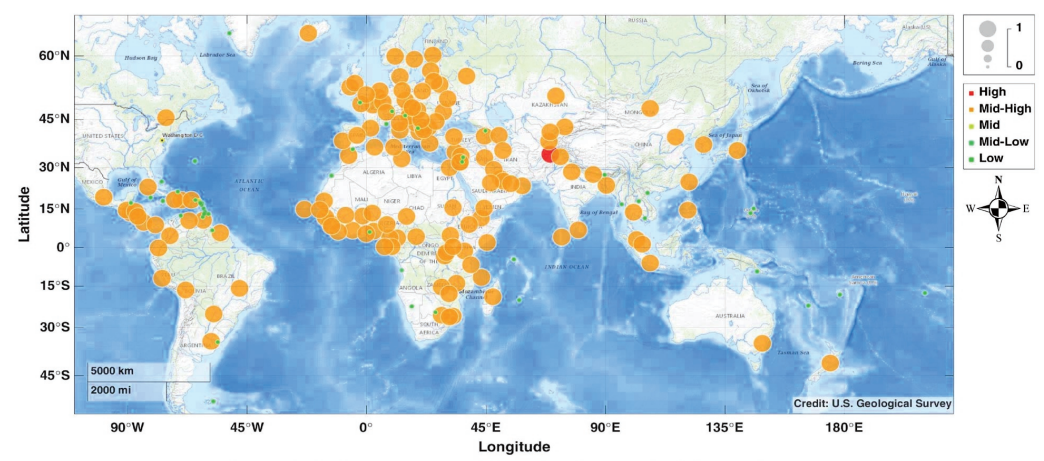

c) The importance of sixty six-days period for each country

\section{FIGURE 17.8}

The important periods of the COVID-19 with their magnitude.

There have been periods of 3 and 66 days in many countries, but the magnitude of three days periods was much greater than 66 days. Periods of six days have been observed in many parts of Europe, some parts of Africa, and South America. Neighboring countries may have an impact on the similarity of the importance of periods. 
In the COVID-19 prediction for each country, the performance of the proposed model was investigated in different situations based on the test and train RMSE, and the DWT and CWT mother wavelet functions were determined as well as an optimal network was built. Since train data was larger than test data and there had been significant changes and sudden increases in the train data, test accuracy was higher than train accuracy in many countries. Therefore, the train data was much more irregular than the test data.

Due to the different populations, confirmed cases and periods of infection in different countries, the RMSE is not sufficient to compare the accuracy of prediction for various countries. RMSEIQR can be more compatible to compare different data sets through IQR normalization. It has been determined for train and test data in all countries and the best and worst values are shown in Figure 17.9. According to the proposed model and the train data, Russia with RMSEIQR of 0.018 was the best, and Mauritania with 54.2689 was the worst prediction of this study.
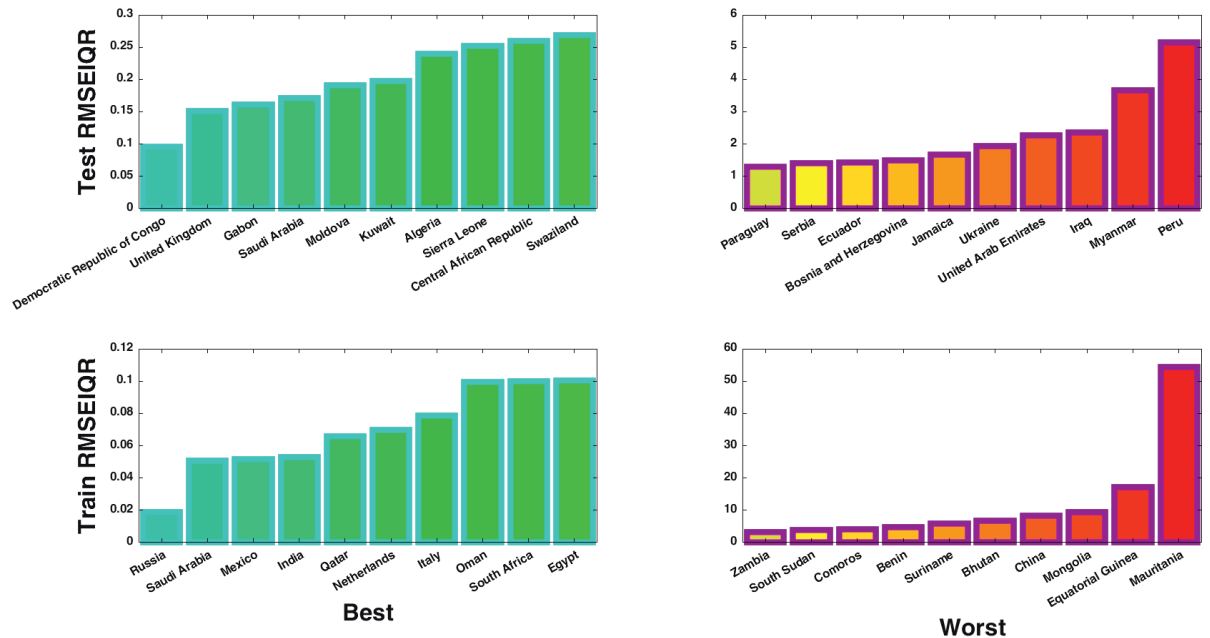

FIGURE 17.9

Comparison of test and train RMSEIQR.

Based on the test data, the Democratic Republic of Congo with RMSEIQR of 0.095 was the best, and Peru with 5.14 was the worst. The results indicate that the performance of the prediction was sufficient for almost all countries. In particular, the networks of countries such as Russia, Mexico, Italy, the United Kingdom, India, Saudi Arabia, and the Congo were the most efficient.

\subsection{Conclusion}

In this chapter, daily reports of the COVID-19 cases through different countries of the world were used to discover important periods of the disease. The results indicate that the COVID-19 has different short-term and long-term periods in different countries of the world, but in general, the main important periods were between 2 and 7 days, which had been demonstrated by clinical study for limited countries. Significant long periods, such as 66 days and 41 days, have also been detected by CWT. Then, by DWT, the features were extracted and important periods were used as a delay on the main signal and the extracted features. The neural network was then built based on the results of the previous steps and the COVID-19 cases were predicted. Results were acceptable with an average train and test RMSEIQR equivalent to 1.2785 and 0.6695 , respectively. 
Finally, hot/cold spots were identified by analyzing spatiotemporal patterns. The existence of $48.78 \%$ and $13.20 \%$ of oscillation and consecutive hot spot patterns around the world indicate the widespread spatiotemporal distribution of this epidemic. Prediction and analysis of hot/cold spots are useful for finding high-risk areas. Accurate predictions of cases and deaths can help politicians and decision-makers to legislate until the COVID-19 vaccine is discovered. A comparison of various neural networks and their tunes with optimization methods may be discussed in future research. Also, effective environmental and social factors in the COVID-19 and the impact of neighboring countries will be extracted which are recommended for further study in this field.

\section{References}

[1] Novel Coronavirus (2019-nCoV) situation reports - World Health Organization (WHO), https://covid19.who.int [accessed on 5 October 2020].

[2] Lai, Ch. Ch., Shih, T. P., Ko, W. Ch., Tang, H. J., Hsueh, P. R., 2020. Severe acute respiratory syndrome coronavirus 2 (SARS-CoV-2) and coronavirus disease-2019 (COVID-19): The epidemic and the challenges. International Journal of Antimicrobial Agents, 55, 105924. doi: 10.1016/j.ijantimicag.2020.105924.

[3] Celso A. G. Santos, C. A. G., Freire, P. K. M. M., Akrami, S. A., Silva, R. M. D., 2019. Hybrid Wavelet Neural Network Approach for Daily Inflow Forecasting Using Tropical Rainfall Measuring Mission Data. Journal of Hydrologic Engineering 24(2). doi: 10.1061/(ASCE)HE.1943-5584.0001725.

[4] Al-Ahmadi, Kh., Alahmadi, S., Al-Zahrani, A., 2019. Spatiotemporal Clustering of Middle East Respiratory Syndrome Coronavirus (MERS-CoV) Incidence in Saudi Arabia, 2012-2019. International Journal of Environmental Research and Public Health, 16(14), 1-14. doi:10.3390/ijerph16142520.

[5] Mongkolsawat, Ch., Kamchai, T., 2008. GIS Modeling for Avian Influenza Risk Areas, 2nd international conference on HealthGIS, Bangkok, 14-16 January.

[6] Li, Z., Fu, J., Lin, G., Jiang, D., 2019. Spatiotemporal Variation and Hotspot Detection of the Avian Influenza A(H7N9) Virus in China, 2013-2017. International Journal of Environmental Research and Public Health, 16, 648; doi:10.3390/ijerph16040648.

[7] Zhu, X., Fu, B., Yang, Y., Ma, Y., Hao, J., Chen, S., Liu, Sh., Li, T., Liu, S., Guo, W., Liao, Zh., 2019. Attention-based recurrent neural network for influenza epidemic prediction. BMC Bioinformatics, 20(18), 575. doi: 10.1186/s12859-019-3131-8.

[8] Venna, S.R., Tavanaei, A., Gottumukkala, R. N., Raghavan, V. V., Maida, A. S., Nichols, S., 2019. A novel data-driven model for real-time influenza forecasting. IEEE Access, 7, 7691-7701. doi: 10.1109/ACCESS.2018.2888585.

[9] Spataru, R., 2018. Spatial-temporal GIS analysis in public health: a case study of polio disease, Master Thesis, Department of Physical Geography and Ecosystem Science, Centre for Geographical Information Systems, Lund University, Sweden.

[10] Guan, W., Ni, Z., Hu, Y., Liang, W., Ou, C., He, J., Liu, L., Shan, H., Lei, C., Hui, D. S. C., Du, B., Li, L., Zeng, G., Yuen, K. Y., Chen, R., Tang, C., Wang, T., Chen, P., Xiang, J., Li, S., Wang, J. L., Liang, Z., Peng, Y., Wei, L., Liu, Y., Hu, Y. H., Peng, P., Wang, J. M., Liu, J., Chen, Z., Li, G., Zheng, Z., Qiu, S., Luo, J., Ye, C., Zhu, S., Zhong, N., 2020. Clinical Characteristics of Coronavirus Disease 2019 in China. The New England Journal of Medicine. doi: 10.1056/NEJMoa2002032.

[11] Kuniya, T., 2020. Prediction of the Epidemic Peak of Coronavirus Disease in Japan, Journal of Clinical Medicine, 9, 789; doi:10.3390/jcm9030789.

[12] Al-qaness, M. A. A., Ewees, A. A., Fan,H ., Aziz, M. A. E., 2020. Optimization method for forecasting confirmed cases of COVID-19 in China. Journal of Clinical Medicine, 9, 674. doi:10.3390/jcm9030674.

[13] Chakraborty, T., Ghosh, I., 2020. Real-time forecasts and risk assessment of novel coronavirus (COVID-19) cases: A data-driven analysis. medRxiv. doi:10.1101/2020.04.09.20059311.

[14] Tamang, S. K., Singh, P. D., Datta, B., 2020. Forecasting of Covid-19 cases based on prediction using artificial neural network curve fitting technique. Global J. Environ. Sci. Manage, 6 (SI): 53-64. doi:10.22034/GJESM.2019.06.SI.06.

[15] Dyllon, Sh., Xiao, P., 2018. Wavelet Transform for Educational Network Data Traffic Analysis. Wavelet Theory and Its Applications, IntechOpen,eBook (PDF) ISBN: 978-1-83881-536-3. doi: 10.5772/intechopen.76455. 
[16] Nourani Esfetanaj, N., Nojavan, S., 2018. The Use of Hybrid Neural Networks, Wavelet Transform and Heuristic Algorithm of WIPSO in Smart Grids to Improve Short-Term Prediction of Load, Solar Power, and Wind Energy. Chapter 4, Operation of Distributed Energy Resources in Smart Distribution Networks. doi: 10.1016/B978-0-12-814891-4.00004-7.

[17] Conejo, A.J., Plazas, M., Espinola, R., Molina, A.B., 2005. Day-ahead electricity price forecasting using the wavelet transform and ARIMA models, Power Systems, IEEE Trans, 20, 1035-1042. doi: 10.1109/TPWRS.2005.846054.

[18] Ahmed. O., Ovinis, M., Hashim, F., 2017. Osei, H. Time Delay Estimation Using Continuous Wavelet Transform Coefficients. Journal of Computational and Theoretical Nanoscience 23(2):1299-1303. doi: $10.1166 /$ asl.2017.8377.

[19] Farge, M., 1992. Wavelet Transforms and Their Application to Turbulence. Ann. Rev. Fluid. Mech., 24, 395-457. doi: 10.1146/annurev.fl.24.010192.002143.

[20] Lilly, J. M., 2016. jLab: A data analysis package for Matlab, version 1.6.2., http://www.jmlilly.net/jmlsoft.html [accessed on 8 July 2020].

[21] Chaovalit, P., Gangopadhyay, A., Karabatis, G., Chen, Zh., 2011. Discrete wavelet transform-based time series analysis and mining. ACM Computing Surveys, 43(2), 1-37. doi: 10.1145/1883612.1883613.

[22] Merry, R. J. E., 2005. Wavelet theory and applications: a literature study. (DCT rapporten; Vol. 2005.053).

[23] Rosenblatt, F., 1961. Principles of Neurodynamics: Perceptrons and the Theory of Brain Mechanisms. Spartan Books, Washington DC

[24] Klimasauskas, C. C., 1991. Applying neural networks, part III: Training a neural network. PC-AI, 20-24.

[25] Li, L., Girguis, M., Lurmann, F., Wu, J., Urman, R., Rappaport, E., Ritz, B., Franklin, M., Breton, C., Gilliland, F., Habre, R., 2019. Cluster-based bagging of constrained mixed-effects models for high spatiotemporal resolution nitrogen oxides prediction over large regions. Environment International, 128, 310-323.doi: 10.1016/j.envint.2019.04.057.

[26] Zeng D, Chang W, Chen H., 2004. A comparative study of Spatio-temporal hotspot analysis techniques in security informatics. The 7th International IEEE Conference on Intelligent Transportation Systems, doi: 10.1109/ITSC.2004.1398880.

[27] NetCDF Home Page,https://www.unidata.ucar.edu/software/netcdf/ [accessed on 8 July 2020].

[28] ESRI, 2016, ArcGIS Resources Center. http://resources.esri.com/help/10.1/ArcGISEngine/java GpToolRef/Spatial Statistics tools/how hot spot analysis colon getis ord gi star spatial statistics works.html [accessed on 26 March 2020].

[29] Abdulhafedh A., 2017. A Novel Hybrid Method for Measuring the Spatial Autocorrelation of Vehicular Crashes: Combining Moran's Index and Getis-Ord Gi* Statistic. Open Journal of Civil Engineering, 7, 208-221. doi:10.4236/ojce.2017.72013.

[30] Qun Li et al., 2020. Early Transmission Dynamics in Wuhan, China, of Novel Coronavirus-Infected Pneumonia - New England Journal of Medicine, 382(13), 1-9. doi: 10.1056/NEJMoa2001316.

[31] Bai, Y., Yao, L., , Wei, T., Tian, F., Jin, D. Y., Chen, L., Wang, M., 2020. Presumed Asymptomatic Carrier Transmission of COVID-19. JAMA, 323(14), 1406-1407. doi: 10.1056/NEJMoa2001316. 